Questions vives

\section{Questions Vives}

Recherches en éducation

$\mathbf{N}^{\circ} 24 \mid 2015$

Accompagnement des transitions professionnelles et dispositifs réflexifs en formation initiale et continue

\title{
Accompagnement des transitions professionnelles et dispositifs réflexifs en formation initiale et continue
}

Thérèse Pérez-Roux

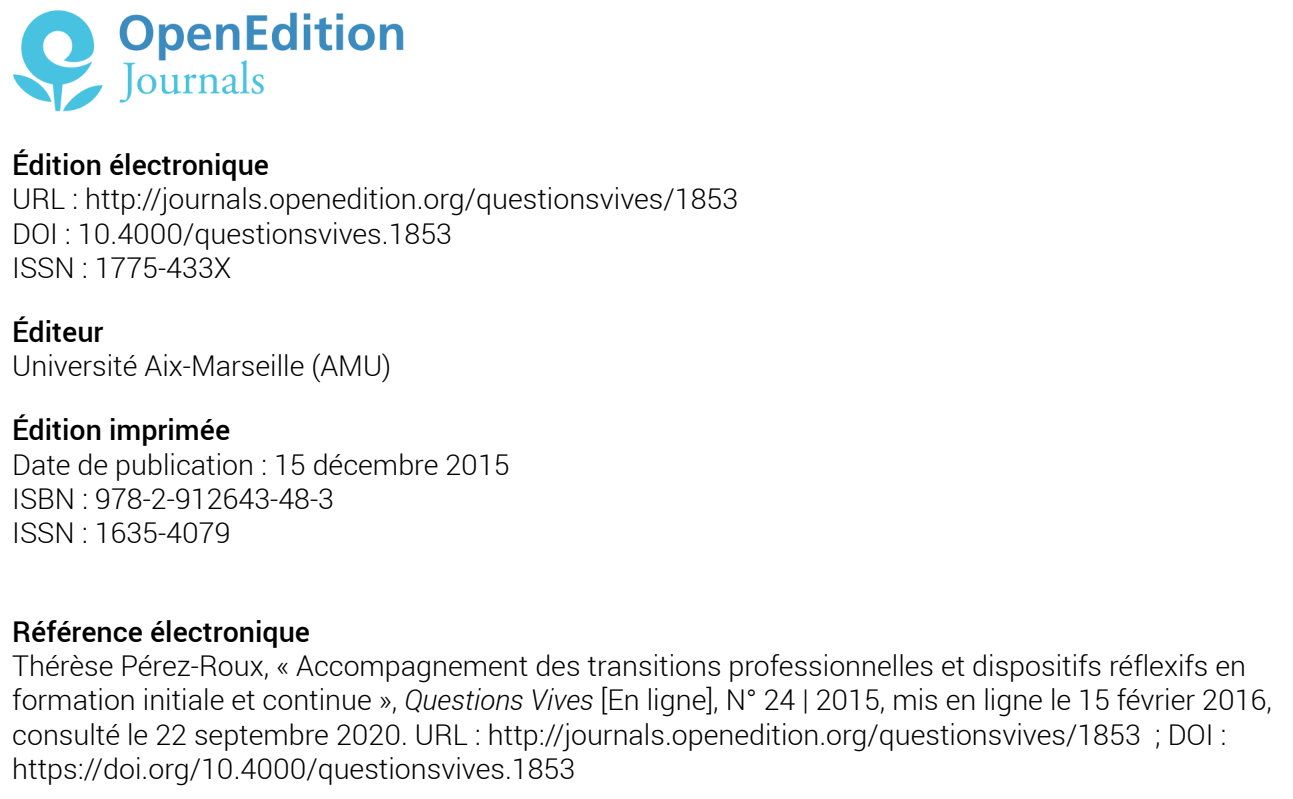

Ce document a été généré automatiquement le 22 septembre 2020.

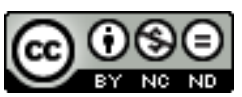

Questions Vives est mis à disposition selon les termes de la licence Creative Commons Attribution Pas d'Utilisation Commerciale - Pas de Modification 4.0 International. 


\title{
Accompagnement des transitions professionnelles et dispositifs réflexifs en formation initiale et continue
}

\author{
Thérèse Pérez-Roux
}

1 La plupart des sociétés occidentales connaissent actuellement des évolutions majeures qui impactent les modalités de formation dans un ensemble de métiers à forte dimension relationnelle (enseignement, éducation, santé, travail social). Ces transformations nécessitent la mise en place de dispositifs renouvelés pour accompagner des périodes de transition plus ou moins désirées par les acteurs. Le plus souvent, le changement est associé à des remaniements identitaires liés à divers facteurs : perte de repères antérieurs, difficulté d'intégration dans les nouvelles normes institutionnelles ou organisationnelles, sentiment de (non)reconnaissance des compétences construites, etc. (Balleux \& Perez-Roux, 2011).

2 Les périodes de transition peuvent générer chez les adultes des formes de fragilité et de vulnérabilité. Pour gérer le changement dans des environnements complexes et déstabilisants, le recours à un accompagnement devient nécessaire, voire indispensable (Boutinet, 2009). Or, si toutes les formes d'accompagnement tendent à se définir comme pratiques intégratives et temporaires (Paul, 2009), visant à favoriser une dynamique de changement, elles peuvent aussi concerner des groupes en formation. L'accompagnement s'adosse alors à un dispositif, le plus souvent réflexif.

Dans la triangulation formateur-formé(s)-dispositif, plusieurs processus sont activés :

- un processus de subjectivation, entendu comme une appropriation subjective du réel, entre émancipation et assujettissement (Martuccelli, 2005);

- un processus de responsabilisation qui, dans les "métiers de l'humain», suppose de se confronter à la fois à l'incertitude des choix que l'on est amené à faire et à l'obligation d'en assumer les conséquences (Jaeger, 2009); 
- un processus d'acculturation entendu comme l'adaptation d'un individu ou d'un groupe à la (nouvelle) sphère professionnelle : appropriation des normes à la fois explicites et implicites du milieu, compréhension des limites réelles ou symboliques que se définit le groupe professionnel, etc.

Les différentes contributions de ce numéro thématique abordent les transitions professionnelles à partir d'une perspective interactionniste dans laquelle l'individu est considéré comme participant de façon active au changement social : il utilise donc la transition pour faire des choix dans un environnement qui se transforme. Saisissant contraintes et opportunités du contexte, il décide, s'engage, se met en projet. La transition est alors envisagée comme une chance de développement de la carrière ou comme moyen de renforcer/retrouver ses valeurs, comme une " phase de reconstruction active des valeurs et des normes fondant la reconnaissance et la valorisation de soi et autrui » (Dupuy \& Le Blanc, 2001, p. 68). Cette approche prend en compte la responsabilité du sujet dans ce processus, amenant à considérer les transitions « comme des espaces-temps de co-construction du changement individuel et social » (p. 74) dans lesquels les trajectoires socio-professionnelles sont liées à la fois aux contextes socio-économiques et culturels mais aussi aux stratégies singulières intégrant représentations, valeurs, rôles et modes d'implication professionnelle (Perez-Roux, 2011).

Dupuy et Le Blanc (2001) ont souligné le caractère hétérogène des situations de transition: celles-ci supposent moments de déstabilisation et de relative stabilité, continuité et rupture, changement et résistance au changement. Pris dans cette dynamique, les individus et les groupes développent des stratégies de régulation et d'adaptation. Enfin, par la combinaison de cadres de référence, de modes de pensée et d'action anciens et nouveaux, la transition génère des formes de remaniement identitaire (Perez-Roux, 2016).

Dans ce numéro, les transitions professionnelles sont abordées dans leur dimension à la fois spatiale et temporelle. En effet, dans le cadre de la formation initiale et continue des enseignants et des formateurs, nous nous intéressons à l'accompagnement de diverses situations de transition professionnelle liées :

-à des formations par alternance (master de l'enseignement et de la formation en France, formation au bac en enseignement au Québec, etc.) pour lesquelles les appropriations de savoirs pluriels et composites se révèlent problématiques ;

- à des changements de missions ou de fonctions dans un espace professionnel qui nécessite la construction de nouvelles compétences mais aussi la prise en compte d'enjeux et de logiques parfois concurrentes ;

- à des transformations structurelles de la formation qui rendent perplexes des acteurs supposés s'adapter à de nouveaux modèles de professionnalisation.

7 L'enjeu de ce numéro consiste aussi à interroger, à l'échelle internationale, les moyens mis en place pour accompagner ces transitions, et plus particulièrement les dispositifs à visée réflexive étudiés par la recherche en éducation. La notion de dispositif est entendue ici comme un outil opérationnel de régulation, relativement malléable et visant une efficacité pratique (Fusulier \& Lannoy, 1999). Nous retiendrons les trois invariants du dispositif mis en avant par Maleyrot (2012) pour éclairer les différentes contributions. Le dispositif est d'abord une organisation sociotechnique de moyens dans un environnement spécifique au service d'une stratégie, d'une action finalisée, planifiée qui vise un résultat précis. Il s'inscrit dans une volonté de maîtrise du réel et introduit une dynamique plus proche des réalités rencontrées par les acteurs dans leur 
pratique. Enfin, un dispositif n'est jamais totalement normalisable, consensuel, réalisé comme il a été prévu: traversé par des tensions entre idéel, fonctionnel et vécu (Albero, 2010), il suppose des modes d'appropriation singuliers et des choix de la part des différents acteurs concernés.

En ce sens, une approche par les dispositifs et leurs effets dans les périodes de transition peut engager plusieurs questions :

- comment et dans quelle mesure ces dispositifs à visée réflexive aident-ils les (néo)professionels à construire une (nouvelle) professionnalité ?

- quelles sont les difficultés de mise en œuvre et les conditions de possibilité pour qu'ils produisent les effets attendus?

- quelles questions transversales cela pose-t-il au plan des compétences professionnelles, des registres de savoirs et des constructions (ou des dynamiques) identitaires?

- quelles pistes de transférabilité émergent de ces recherches internationales et quels appuis pour la formation universitaire et/ou professionnelle?

9 Le numéro thématique est structuré autour de quatre entrées choisies à partir des types de formation (initiale et continue) et des publics ciblés: étudiants (dans différents pays, secteurs et systèmes de formation), enseignants (français, québécois, chiliens) et formateurs.

a première partie s'intéresse aux dispositifs d'accompagnement, à visée professionnalisante, mobilisés en formation initiale. Cette partie réunit quatre contributions.

11 Nathalie Gal-Petitfaux (université de Clermont-Ferrand, France) analyse l'effet d'un dispositif de vidéo-formation sur la réflexivité avec des étudiants-stagiaires en Éducation physique. A partir du travail en classe de l'enseignant, il s'agit de comprendre comment le dispositif multimodal, intégrant des vidéos en classe et des entretiens d'auto-confrontation avec les enseignants observés sur la vidéo, permet aux étudiants d'approcher l'action en classe en tant qu'expérience corporelle et habiletés d'enseignement. Les résultats montrent dans quelle mesure les étudiants s'approprient le dispositif pour : faire une analyse des habiletés corporelles pour enseigner; se créer une immersion sensorielle dans la classe; transformer leur attention perceptive et envisager de nouvelles façons d'agir en classe.

cadre de l'enseignement vétérinaire, Sylvain Dernat et Arnaud Siméone (université Lyon 2, France) s'intéressent à la politique de formation de ce secteur, qui considère les stages comme un outil majeur de découverte du monde professionnel pour les étudiants. Au travers d'une étude qualitative par entretiens $(n=72)$, les auteurs mettent en lumière la façon dont les stages participent à l'évolution des représentations initiales des étudiants et peuvent les aider dans leur passage vers l'emploi. Ils soulignent aussi certains écueils. L'ingénierie de formation est alors envisagée comme un outil qui favorise la mise en place d'un accompagnement pédagogique tout au long du cursus par des approches participatives et la création d'un véritable espace de médiation de la transition école-travail.

La contribution de Gaëtan Temperman, Joachim De Stercke et Bruno De Lièvre (université de Mons, Belgique) tente de mesurer l'impact d'un dispositif de formation hybride (présentiel et à distance) dont le but est d'aider de futurs enseignants $(n=28)$ d'une haute école pédagogique à s'approprier l'usage du tableau noir au cours de leurs stages sur le terrain. L'idée mobilisatrice de ce projet de formation consiste à amener 
les formés vers l'adoption d'une démarche réflexive avant, pendant et après l'acte d'enseignement. Les auteurs rendent compte d'une évolution des représentations sur l'usage du tableau noir entre les deux stages programmés sur l'année. Considéré d'abord comme un outil d'organisation, le tableau noir est progressivement envisagé comme un média permettant de construire des connaissances. En ce qui concerne la capacité de transfert du dispositif, l'analyse de contenu catégorielle des commentaires des maîtres de stage, dans les carnets de bord, permet de mettre en évidence des progrès chez les futurs enseignants dans cet apprentissage.

Enfin, l'article de Céline Avenel (université de Montpellier 3, France) s'intéresse au monde de la santé et analyse les transitions identitaires dès les premières années de formation en études de médecine, en mettant la focale sur le tutorat comme dispositif d'accompagnement. A partir d'une enquête par suivi longitudinal d'étudiants, l'auteure montre la complexité des phases de passage en examinant les évolutions de statut des étudiants et leur lien avec la socialisation communautaire. Le parcours de formation est ainsi analysé sur la base de rites socialisateurs qui conditionnent l'entrée dans le métier et le rapport au groupe professionnel.

La seconde partie du numéro porte sur des dispositifs de formation à visée intégrative, fortement articulés à l'expérience, c'est-à-dire dans lesquels un tissage doit s'opérer, à travers le dispositif proposé, entre expérience actuelle et passée, entre projection de soi dans le métier et retour réflexif sur l'expérience. Cette partie réunit deux contributions qui proposent des dispositifs à l'interface de la formation initiale et de la formation continue.

16 L'accompagnement des enseignants débutants de l'enseignement professionnel au Québec est abordé par André Balleux et Claudia Gagnon (université de Sherbrooke, Québec, Canada). Ces enseignants, anciens professionnels ayant acquis une expertise dans leur domaine, vivent une transition professionnelle, durant laquelle ils doivent relever de nombreux défis. Etre ou ne plus être le professionnel de son métier en devenant enseignant interroge la légitimité de la transmission des savoirs et des pratiques. Il s'agit de comprendre dans quelle mesure les professionnels devenus étudiants peuvent se sentir encore les héritiers et les transmetteurs d'un métier qu'ils ne pratiquent plus. L'étude s'appuie sur un corpus issu d'un cours dispensé dans le cadre de la formation à l'enseignement qui a pour but d'accompagner une réflexion individuelle et collective sur la transmission d'une culture.

De son côté, Mauricio Nunez-Rojas (université du Chili) propose une recherche collaborative médiatisée par une situation réflexive. Dans le cadre d'un atelier d'écriture de cas, un groupe constitué par des étudiants stagiaires et leurs tuteurs de stage, forme une communauté de pratique. En rapprochant les itinéraires de formation des enseignants débutants avec ceux d'enseignants expérimentés en charge de les accompagner, le dispositif mis en place autour de l'écriture de cas suppose la coconstruction d'une expérience professionnelle, individuelle et collective. L'étude met en lumière le potentiel de transformation généré par ce dispositif qui soutient et favorise le processus de professionnalisation des enseignants concernés.

La troisième partie du numéro, qui réunit trois contributions, concerne des dispositifs de formation continue mis en place pour accompagner/favoriser le développement professionnel des acteurs.

19 Cécilia Borges et Colette Gervais (université de Montréal, Québec, Canada) s'intéressent à l'accompagnement d'enseignants à partir d'un dispositif d'analyse de pratiques 
inspiré de l'approche de «l'argumentation pratique ». L'étude porte sur 13 enseignants inscrits dans un cours à l'université. Les auteures s'appuient sur des productions écrites résultant du travail d'analyse réalisé dans le dispositif; celui-ci s'inscrit dans une perspective de développement professionnel. Cinq dimensions sont mises en lumière à partir des bilans: le rapport au savoir, la vision de l'enseignement, l'identité professionnelle, le développement professionnel, le rapport au collectif.

Le dispositif de co-développement professionnel présenté par Joëlle Morrissette et Yasmine Charara (université de Montréal, Québec, Canada) a pour but de soutenir les enseignants en emploi dans ce qu'ils vivent comme étant une période de transition professionnelle. Quatre enseignants d'une école secondaire située sur l'île de Montréal ont été accompagnés. Les auteures analysent les récits de pratique contrastés de deux de ces enseignants, marqués par les ressources discursives des membres du groupe qui mettaient à leur disposition leurs expertises variées. Dans un second temps, elles examinent dans quelle mesure ce dispositif constitue un levier de réflexivité et tentent d'en cerner les éventuels écueils.

21 La contribution de Thérèse Pérez-Roux (Université de Montpellier 3, France) s'intéresse à l'accompagnement de nouveaux acteurs de l'éducation : les enseignants-référents ( $n$ =15) pour l'Adaptation scolaire et la Scolarisation des élèves Handicapés qui ont à assumer la transition d'une fonction vers une autre. Cette transition est soutenue par un dispositif d'analyse de pratiques. Les résultats mettent en avant de nombreuses tensions. Au-delà de la distanciation affective à laquelle les invite l'institution, les enseignants-référents évoquent l'impact de la dimension relationnelle sur leur activité. Le dispositif d'accompagnement est perçu par ces acteurs comme une aide à la transition et l'occasion de co-construire, sur la base de situations emblématiques (partagées), les prémisses d'un « genre professionnel ».

22 La quatrième partie du numéro, orientée vers les dispositifs d'accompagnement en formation de formateurs, réunit trois contributions.

23 Tout d'abord, l'étude de Nicole Clerc et Martine Agogué (université de Cergy-Pontoise, France) concerne les professionnels des «métiers de la relation». Elle cherche à identifier les processus de remaniements identitaires des formateurs, concernés dans leurs missions par les impacts contemporains des mutations sociétales. Une vignette clinique d'une séance d'analyse réflexive des pratiques permet d'étudier comment le temps de la «méta-analyse», «espace transitionnel» contenant, facilite la compréhension des processus psychiques liés aux prises de conscience et aux nécessaires déplacements des représentations. Les modalités de travail décrites et analysées selon un principe d'association, dans une logique de contamination visant la transmission du geste partagé, ouvrent l'accès à une pensée suspendue et engagée. À partir des témoignages des participants, les auteures identifient les ressources créatives de cet espace transitionnel: d'une part, il renouvelle le pouvoir de comprendre et d'agir ; d'autre part, il permet au sujet démocratique d'advenir dans un espace collectif du " penser ensemble ».

Dans un autre contexte, Vincent Grosstephan (Université de Reims, France) rend compte des conséquences de la mastérisation de la formation des enseignants sur le travail des formateurs d'un Institut Universitaire de Formation des Maîtres (IUFM). Audelà du diagnostic établi en vue de l'élaboration d'un plan de formation continue, l'auteur tente, à partir de l'analyse de situations de travail, de mettre au jour la façon dont les formateurs de cet IUFM perçoivent et vivent les conséquences de cette 
réforme. Cette dernière est abordée comme un moment de transition institutionnelle et professionnelle particulier, qui affecte la professionnalité des différents acteurs. L'étude montre dans quelle mesure le dispositif réflexif, support du recueil de données, permet de créer les conditions d'une réappropriation individuelle et collective du métier par les formateurs.

Enfin, la contribution d'Eric Maleyrot (université de Nantes, France) se centre sur un groupe de formateurs d'enseignants de l'école primaire. L'étude a pour objectif de dégager, à travers les missions des maîtres formateurs ces dernières années, les modèles de professionnalité et les compétences valorisées pour accompagner les transitions professionnelles des enseignants du premier degré. L'auteur cherche également à déterminer la nature de la visée réflexive des dispositifs d'accompagnement à l'entrée dans le métier, au regard des activités de formation des maîtres formateurs. L'analyse dégage trois figures de professionnalité successivement valorisées pour former les futurs enseignants; elle souligne les évolutions et les invariances de ces dispositifs d'accompagnement ainsi que les limites à l'aide réflexive apportée par les maîtres formateurs dans le cadre de leurs missions.

\section{BIBLIOGRAPHIE}

Albero, B. (2010). La formation en tant que dispositif : du terme au concept. In B. Charlier, F. Henri, (Eds.), La technologie de l'éducation : recherches, pratiques et perspectives (pp. 47-59). Paris : Presses Universitaires de France.

Balleux, A \& Perez-Roux T. (2011, sdr). Transitions professionnelles et recompositions identitaires dans les métiers de l'enseignement et de l'éducation. Recherches en éducation, 11. http://www.recherches-en-education.net/IMG/pdf/REE-no11.pdf

Boutinet, J-P. (2009). Entretien. Recherche et formation, 62, 109-123.

Dupuy, R \& Le Blanc, A. (2001). Enjeux axiologiques et activités de personnalisation dans les transitions professionnelles. Connexions, 2001/2, 76, 61-79.

Fusulier, B. \& Lannoy, P. (1999). Comment « aménager par le management », Hermès, 25,181-198. Jaeger, M. (2009). Du principe de responsabilité au processus de responsabilisation. Vie sociale, 3, 71-81.

Maleyrot, E. (2012). Évolution de la fonction et de l'identité professionnelle des maîtres formateurs. Thèse de doctorat en Sciences de l'Éducation, Université de Nantes, non publiée.

Martuccelli, D. (2005). Les trois voies de l'individu sociologique. EspacesTemps.net, http:// www.espacestemps.net/articles/trois-voies-individu-sociologique

Paul, M. (2009). Autour du mot accompagnement. Recherche et formation, 62, 91-107.

Perez-Roux, T. (2011). Identité(s) professionnelle(s) des enseignants : les professeurs d'EPS entre appartenance et singularité. Paris : Éditions EP.S. 
Perez-Roux, T. (2016). Transitions professionnelles et transactions identitaires : expériences, épreuves, ouvertures. Pensée plurielle, 41 (1), 81-93.

AUTEUR

THÉRÈSE PÉREZ-ROUX

LIRDEF EA 3749, Université Paul Valéry 